\title{
Executive function and its relation to anatomical connectome in homosexual and heterosexual men
}

\author{
Zheng Wang ${ }^{1,2}$, Jian Bo Hu ${ }^{1,2}$, Gong Jun $\mathrm{Ji}^{3}$, Dong Rong $\mathrm{Xu}^{4}$, Dan Dan Wang ${ }^{1,2}$, Cai Xi Xi, \\ Chan Chan $\mathrm{Hu}^{1,2}$, Jing Lu ${ }^{1,2}$, Yan Li Du ${ }^{5}$, Qiao Qiao Lu ${ }^{5}$, Ting Ting Huang ${ }^{5}$, Jian Bo Lai, \\ Jing Kai Chen ${ }^{1,2}$, Wei Hua Zhou ${ }^{1,2}$, Ning Wei ${ }^{1,2}$, Yi Xu ${ }^{1,2}$, Kai Wang ${ }^{3}$, Shao Hua Hu ${ }^{1,2}$ \\ ${ }^{1}$ Department of Psychiatry, First Affiliated Hospital, Zhejiang University School of Medicine, Hangzhou, China; ${ }^{2}$ The Key Laboratory of Mental \\ Disorder's Management of Zhejiang Province, Hangzhou, China; ${ }^{3}$ Laboratory of Cognitive Neuropsychology, Department of Medical Psychology, \\ Anhui Medical University, Hefei, China; ${ }^{4}$ Epidemiology Division \& MRI Unit, Department of Psychiatry, Columbia University \& New York State \\ Psychiatric Institute, New York, NY, USA; ${ }^{5}$ Zhejiang University School of Medicine, Hangzhou, China
}

Correspondence to: Shao Hua Hu, MD. Department of Psychiatry, First Affiliated Hospital, Zhejiang University School of Medicine, Hangzhou 310000, China; The Key Laboratory of Mental Disorder's Management of Zhejiang Province, Hangzhou 310000 , China. Email: dorhushaohua@zju.edu.cn.

Background: Sexual orientation has been suggested to affect executive function, of which the neurobiological basis is still largely unknown. In this study, we explored the interrelationship between neuropsychological characteristics in homosexual and heterosexual men and their anatomical connectome by graph theoretical analysis.

Methods: Fifty-three homosexual and 47 heterosexual males underwent diffusion tensor magnetic resonance imaging (MRI) and neuropsychological assessments. Whole-brain anatomical networks were constructed using white matter tractography, performed on the diffusion tensor imaging data. Neuropsychological tests included the Wisconsin Card Sorting Test (WCST), the Continuous Performance Test (CPT) and the Trail-Making Test (TMT).

Results: The cognitive performance of homosexual men was significantly poorer than their heterosexual counterparts in terms of WCST total correct responses. Anatomical connectome analysis revealed a lower $(\mathrm{P}=0.001)$ anatomical connectivity between left PoCG and left SMG $(\mathrm{P}=0.003)$ in homosexual men as compared to heterosexual men. Linear regression analyses showed that the WCST total correct responses score was significantly linked with sexual orientation $(\mathrm{P}=0.001)$. The anatomical connectivity strength between left PoCG and left SMG was also shown to be significantly correlated with sexual orientation $(\mathrm{P}=0.039)$ and education $(\mathrm{P}=0.047)$.

Conclusions: Our study demonstrated the differences in the performance of WCST and anatomical connectome of large-scale brain networks between homosexual and heterosexual men, extending our understanding of the brain's circuitry and the characteristics of executive function in men of different sexual orientation.

Keywords: Homosexual man; neuropsychological characteristic; executive function; magnetic resonance imaging (MRI); anatomical connectome

Submitted Oct 01, 2019. Accepted for publication Jul 14, 2020.

doi: $10.21037 /$ qims-19-821b

View this article at: http://dx.doi.org/10.21037/qims-19-821b 


\section{Introduction}

Executive function is one of the most advanced cognitive functions in humans, involved in handling novel situations outside the domain of some automatic psychological processes that are not explained by the reproduction of learned schemas or set behaviors (1). Smith and Jonides (2) suggested that executive function is comprised of five executive components: attention and inhibition, coding, monitoring, planning, task management. People with normal executive functions can distinguish essential information, implement plans, reason, and solve problems when they deal with novelty. Various factors, such as gender, age and mental disorders, have also been reported as influencing factors of executive function. Additional evidence also suggested that sexual orientation may be related to differences in executive function.

Furthermore, neurocognitive studies have reported the association of specific cognitive profiles with variations in sexual orientation. Earlier studies have shown that heterosexual men, on average, have better visuospatial abilities than homosexual men, whereas profiles for heterosexual women did not differ significantly from homosexual men (3-5). Heterosexual men tended to display superior performance on mental rotation tasks compared to homosexual men. These tasks were typically performed better by heterosexual males compared to heterosexual women (3). Homosexual men, in contrast, had better memory for spatial location and landmarks during navigation, along with superior phonological and semantic fluency, which heterosexual women were known to perform better in, compared to heterosexual men $(5,6)$. However, other studies indicated that no specific cognitive ability differed between heterosexual and homosexual individuals (7). Therefore, the impact of sexual orientation on cognitive abilities is still poorly defined.

The biological basis of human sexual orientation has been hypothesized over the past several decades to include genetic factors (8-10), prenatal androgens (11), maternal immunity (12-14), and neurophysiological factors (14) that determine same-sex attractions over attractions of the opposite sex. Additionally, executive functional differences may be linked to cerebral structural and functional differences associated with sexual orientation. In recent years, increasing neuroimaging studies have emerged to reveal the neural correlates of homosexuality. Functional brain differences have also been reported in homosexual individuals. They include altered functional connectivity of the amygdala in positron emission tomography (PET) scans of cerebral blood flow, more widespread connections from the left amygdala to the right amygdala and the anterior cingulate cortex (15), differences in hypothalamic PET activity in response to antidepressant treatment (fluoxetine) (16), and an altered pattern of brain activity during visually evoked sexual arousal (17-20). These findings, coupled with the anatomical findings aforementioned, may provide substantial evidence for differing anatomical and functional brain phenotypes associated with distinct sexual orientations. However, exploratory investigations into neuroanatomical characteristics with diffusion tensor imaging in homosexual men, to date, has been rarely documented.

Overall, the neurons of the brain constitute a formidably complicated circuitry that supports the segregation and integration of information processing (21). In recent years, the development of graph theory has facilitated a quantitative characterization of various aspects of the brain's topological organization of connectivity (22), including modularity (23), hierarchy, centrality, and the distribution of network hubs (22). This study explored the neuropsychological characteristics of homosexual and heterosexual males. Diffusion tensor imaging data were subsequently collected, and the correlation between brain anatomical connectome and cognitive measures were obtained, based on graph theoretical analysis.

\section{Methods}

\section{Participants}

A group composed of 53 self-reported homosexual men (age $23.96 \pm 4.28$ years, range from 18 to 35 years) was recruited from a local underground club of homosexuality in Hangzhou, China. Forty-seven healthy heterosexual ageand education level-matched controls (age $24.34 \pm 2.88$ years, range from 20 to 34 years) were recruited from local communities.

The inclusion criteria included: (I) physical health with no earlier history of physical disease; (II) male adults aged between 18 to 35 years; (III) right-handedness identified by the Edinburgh Handedness Inventory (EHI). The exclusion criteria included: (I) history of psychiatric or neurological disorders, such as depression, bipolar disorder, head trauma, and epilepsy; (II) history of long-term medications or psychoactive substance abuse, including alcohol use and smoking; (III) history of sexual dysfunction, gender identity disorder, or paraphilia which were excluded by the Utrecht Gender Dysphoria Scale (UGS) and the Multiphasic Sex 
Table 1 Demographic data and sexual preference characteristics of 53 homosexual and 47 heterosexual participants

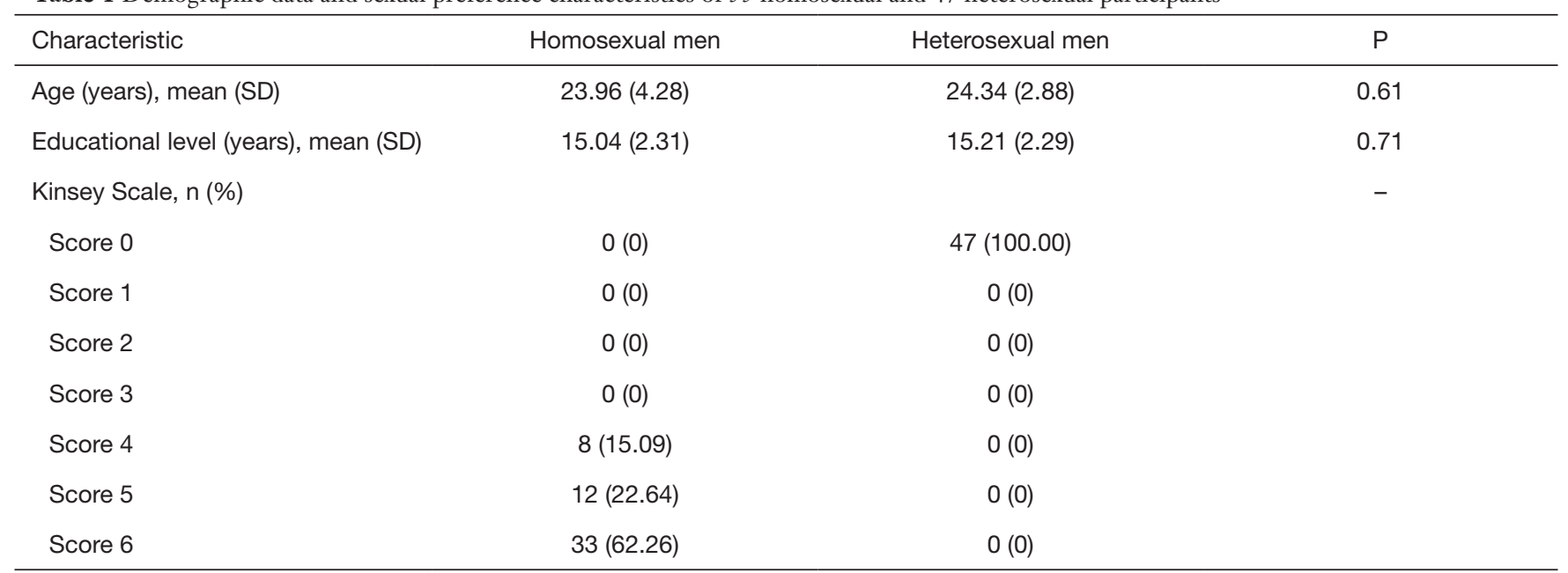

$\mathrm{SD}$, standard deviation.

Inventory (MSI). The sexual preference of each participant was assessed by the Kinsey Heterosexual-Homosexual Rating Scale, which provides an overall index of sexual orientation ranging from 0 (exclusively heterosexual) to 6 (exclusively homosexual). Subjects whose scores ranged from 4 to 6 were recruited as the homosexual group. Participants who scored 0 were assigned as heterosexual (Table 1). All participants were HIV-negative.

The study complied with the Declaration of Helsinki (as revised in 2013) and approved by the ethics committee of the First Affiliated Hospital, Zhejiang University School of Medicine. All participants provided their written informed consent for study participation after the risks and benefits were discussed in full.

\section{Neuropsychological assessments}

The Wisconsin Card Sorting Test (WCST), Continuous Performance Test (CPT), and the Trail-Making Test (TMT) were used to evaluate executive functioning in all participants.

The modified WCST (24) is a widely used psychological assessment instrument for evaluating higher-order cognitive functions related to the frontal lobes (25). Participants were required to assign 48 cards based on color, number, and shape. After six consecutive correct responses, participants were asked to change the sorting principle to another category. The test was finished when participants completed all six categories correctly or assigned all 48 cards. The total trial, correct trials, total number of errors, perseverative errors, random errors, and categories were recorded.

The Conners' CPT measures sustained and selective attention (26). Scores reflect the total number of stimuli, the number of correct targets, omission errors (the number of targets to which the person did not respond), and commission errors (the number of times the person responded to a non-target).

The TMT is a test of visual attention and task switching (27). It consists of two parts where participants are instructed to rapidly and accurately connect a set of 25 dots. In part A, participants were required to quickly connect consecutively numbered circles with lines. In part $\mathrm{B}$, they were asked to alternately combine numbers with assorted colors in ascending order. The time to task completion was measured in seconds.

\section{Data acquisition}

\section{MRI data}

All participants underwent structural and diffusion tensor imaging scanning using a 3.0-Tesla General Electric (GE) Sigma Scanner (Hangzhou, China), with a standard GE quadrature head coil. Participants wore earplugs, and padding (foam cushions) under the head was used to restrict head movement. High-resolution axial T1- and $\mathrm{T} 2$-weighted images were obtained from every participant for the detection of silent lesions. Diffusion tensor images covering the whole brain were obtained using a spin-echo echo planar imaging sequence, including 26 volumes with diffusion gradients applied along 25 non-collinear directions 
( $\mathrm{b}=1,000 \mathrm{~s} / \mathrm{mm}^{2}$ ) and 1 volume without diffusion weighting $\left(\mathrm{b}=0 \mathrm{~s} / \mathrm{mm}^{2}\right)$. Each volume consisted of 46 contiguous axial slices (repetition time $=12,000 \mathrm{~ms}$; echo time $=90 \mathrm{~ms}$; field of view $=120 \times 120 \mathrm{~mm}^{2}$. matrix size $=128 \times 128$; flip angle $=90^{\circ}$; slice thickness $=3 \mathrm{~mm}$; no gap). Subsequently, high-resolution 3D T1-weighted anatomical images were acquired in the sagittal orientation using a magnetizationprepared rapid gradient-echo sequence (repetition time $=7$ $\mathrm{ms}$; echo time $=2.9 \mathrm{~ms}$; field of view $=240 \times 240 \mathrm{~mm}^{2}$, matrix size $=256 \times 256 ; 170$ slices; flip angle $=8^{\circ}$; slice thickness $=1.0$ $\mathrm{mm}$; no gap).

\section{Brain network construction}

\section{Data preprocessing}

Diffusion-weighted images (DWIs) were preprocessed and analyzed using the Pipeline for Analyzing Brain Diffusion Images toolkit (PANDA; http://www.nitrc.org/projects/ panda) (28), which synthesizes procedures in FSL (http://fsl. fmrib.ox.ac.uk/fsl) and the Diffusion Toolkit (DTK, http:// www.trackvis.org/dtk/). Head motion and eddy currentinduced distortion were corrected via the eddy function. The diffusion tensor was subsequently estimated by solving the Stejskal and Tanner equation (29), and the fractional anisotropy (FA) value of each voxel was also calculated using the reconstructed tensor matrix. Finally, whole-brain white matter fiber tracking was performed in the native diffusion space for each participant using the "fiber assignment from the continuous tracking algorithm" embedded in DTK. All tracts were computed by seeding each voxel with an FA greater than 0.2 (30). Path tracking proceeded until either the FA was less than 0.15 , or the angle between the current and the previous path segment was greater than $35^{\circ}(31)$. Fibers of less than $10 \mathrm{~mm}$ or displaying obvious false paths were discarded. As a result, all the fiber pathways in the brain were constructed using the deterministic tractography method.

\section{Anatomical parcellation}

We defined 90 regions of interest (ROIs) according to the Anatomical Automatic Labeling (AAL; http://www.gin.cnrs. fr/AAL) template. The ROIs were transformed into each participant's native diffusion space. Specifically, individual 3D T1 images were coregistered to B0 images and the Montreal Neurologic Institute (MNI) space by unified segmentation in SPM8. These transformation parameters were inverted and applied to the AAL template (31). These ROIs were used as seed and target for fiber tracking.

\section{Connectivity matrix and graph-theoretic measures}

The anatomical connectivity between ROIs $i$ and $j$ was defined as the mean FA of fibers connecting $i$ and $j$. For each participant, the anatomical connectivity matrix " $M$ " had $90 \times 90$ entries, with Aij corresponding to the weighted connectivity between ROI $\mathrm{i}$ and $\mathrm{j}$, also referred to as the link between nodes $i$ and $j$.

Graph theory enables the quantification of network topological properties. Graph measures for each connectivity matrix were calculated using the Brain Connectivity Toolbox (https://sites.google.com/a/ brain-connectivity-toolbox.net/bct/). Small-worldness is an optimal architecture balancing the segregation and integration of information, with similar path length $\left(\lambda=L_{\text {net }}^{w} / L_{\text {random }}^{w} \approx 1\right)$ but higher clustering $\left(\gamma=C_{n e t}^{w} / C_{\text {random }}^{w}>1\right)$ than a random network (32). We evaluated the typical properties of a small-worldness $(\sigma=\gamma / \lambda)$, which is usually a value larger than one. Among several nodal measures in graph theory, we selected connectivity strength to estimate the topological feature of each node due to its high test-retest reliability (33) and clear neurophysiological relevance (34). Nodal strength (Si) was computed as the sum of the weights of all connections of node $\mathrm{i}$, that is as $S_{i}^{w}=\sum_{j \in N} w_{i j}$. This value quantifies the extent to which a node is relevant to the graph.

\section{Statistical analysis}

Sparsity was usually defined as the fraction of the total number of edges remaining in a network. For global and nodal topological characteristics statistics, a single sparsity threshold for connectivity network was preferred by some research groups (35), while a range of sparsity thresholds was used by other investigators $(31,36)$. The latter strategy was adopted in this study for consistency across our previous studies $(36)(0.10 \leq$ sparsity $\leq 0.18$, step $=0.01)$. The minimum sparsity was set so that the average node strength of the threshold network was $2 * \log (\mathrm{N})$, where $\mathrm{N}$ is the number of nodes, and its maximum was set so that the small-worldness of the threshold network was $>1.1$. Further, the area-undercurve was calculated (37) for the global and nodal network metrics, providing an overall estimate for the topological characterization of brain networks, independent of cost threshold. The AUC (i.e., global and nodal properties) and anatomical connectivity (i.e., FA) difference betweengroups were tested using a two-sample t-test with a falsepositive adjustment (34). Pearson's correlation was used to 

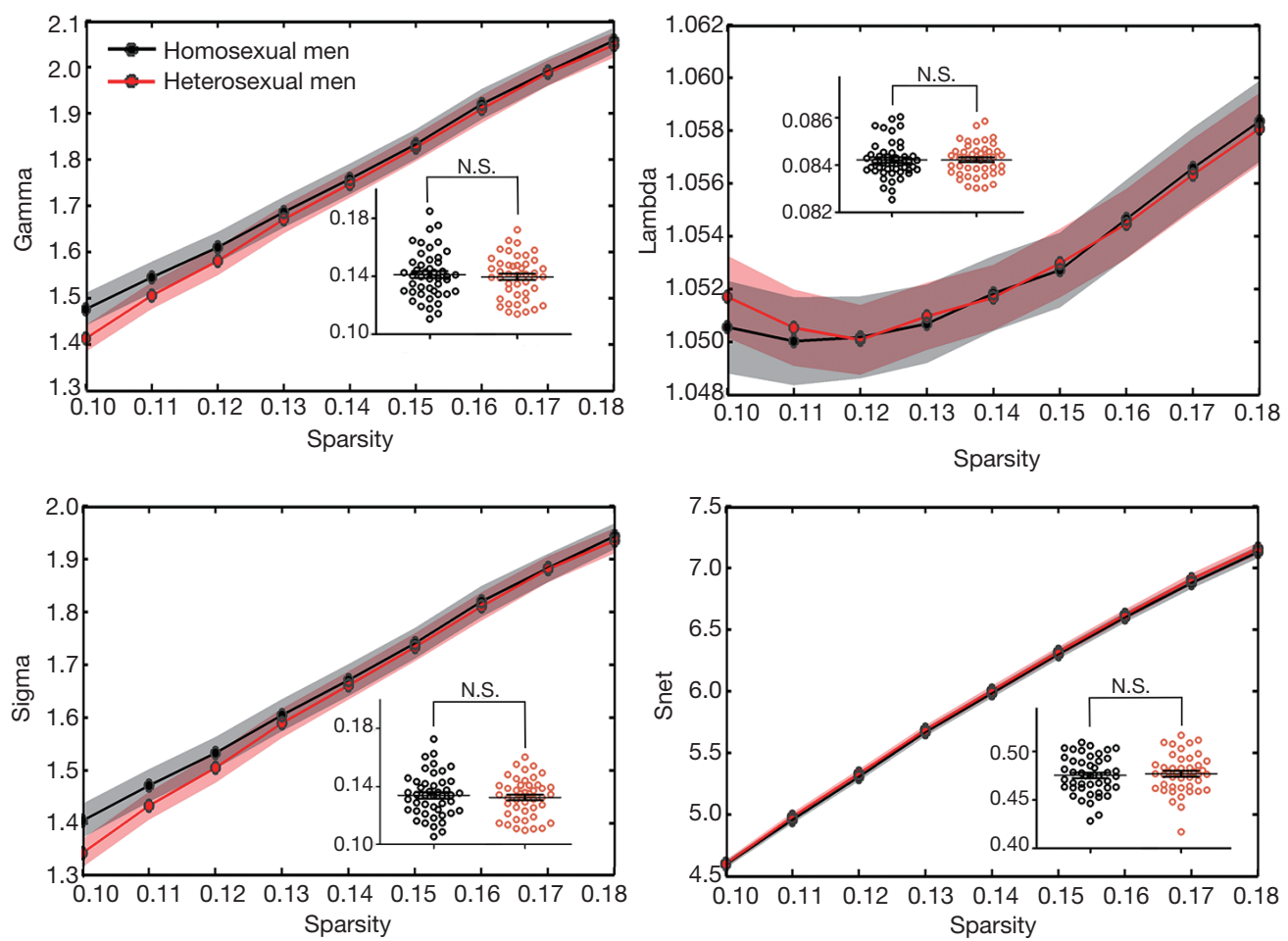

Figure 1 Differences in the topological properties of structural networks between homosexual and heterosexual men. Global metrics of WM structural networks were quantified in two groups with different network sparsity. Shaded bands in black and red denote SEM in homosexual and heterosexual men, respectively. Inset line graphs indicated the between-group analysis of area-under-curve values. N.S., no significance.

analyze the relationship between the topological features (i.e., nodal strength, anatomical connectivity strength) and neuropsychological assessments (i.e., WSCT and CPT scores) in homosexual men. Kolmogorov-Smirnov test was applied to assess the distribution of all neuropsychological test data. The two-sample t-test was used if variables were acceptable with a normal distribution. Alternatively, the Mann-Whitney $U$ test was used if the normal distribution was not achieved. Linear regression was used to estimate the association between neuropsychological assessments, topological features, and sexual orientation, and other clinical characteristics (age and education) separately. The Bonferroni correction was applied to correct for multiple comparisons. A two-sided $\mathrm{P}$ value of less than 0.05 was considered statistically significant. Statistical analyses were performed using SPSS 17.0 software.

\section{Results}

No significant differences were noted between homosexual and heterosexual participants in mean age and education level $(\mathrm{P}>0.05)$. Additionally, 8, 12, and 33 participants from the homosexual group, respectively, scored 4, 5, and 6 on the Kinsey Scale.

\section{Network global features}

A comparison in the anatomical network properties between homosexual and heterosexual men was performed using a data-specific small-world regime within a specified sparsity range $(0.10 \leq$ sparsity $\leq 0.18)$. Both groups exhibited higher clustering coefficients (i.e., $\gamma>1$ ) but almost identical characteristic path length (i.e., $\lambda \approx 1$ ), compared with comparable random networks, which are typical features of small-worldness (Figure 1). No significant difference between groups was observed for global features.

\section{Network nodal and connectivity features}

Anatomical connectivity between the left postcentral gyrus (PoCG) and left supramarginal gyrus (SMG) was lower in homosexual men compared to heterosexual men $(t=-3.12$, 

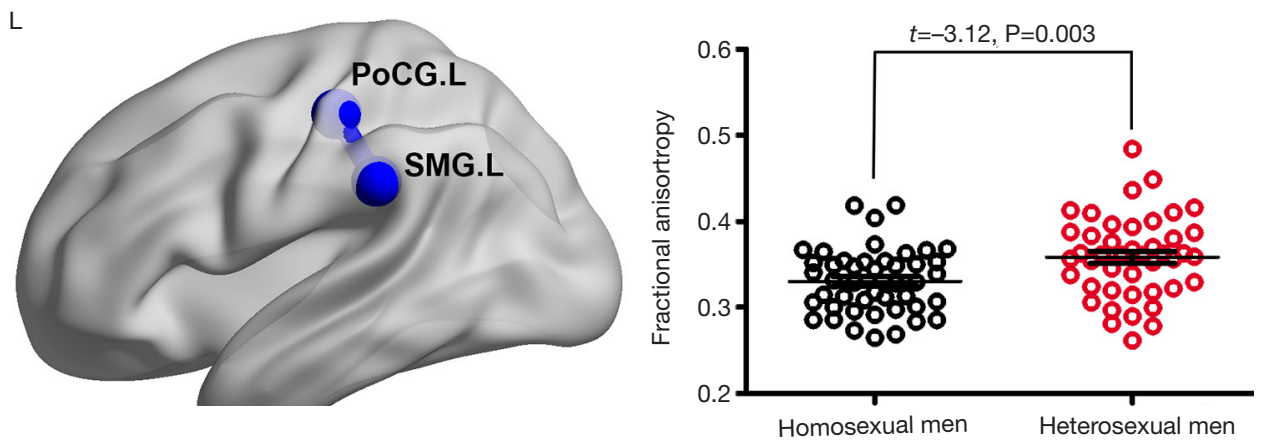

Figure 2 Between-group difference in anatomical connectivity. Decreased connectivity was found between left postcentral gyrus and left supramarginal gyrus in homosexual men compared to heterosexual men. PoCG.L, left postcentral gyrus; SMG.L, left supramarginal gyrus.

uncorrected $\mathrm{P}=0.003$; can survive false-positive adjustment) (Figure 2). There was no significant difference noted on any nodal strength between groups.

\section{Neurocognitive features}

Analysis of data obtained from WCST total correct responses revealed significantly poorer performance by homosexual men compared to heterosexual men $(\mathrm{Z}=-3.36$, uncorrected $\mathrm{P}=0.001$, can survive Bonferroni-corrected). However, both groups did not differ significantly on any of the CPT or TMT performance (Table 2).

\section{Correlation of network characteristics and neurocognitive measures in the homosexual men}

We found no significant correlation between nodal strength or anatomical connectivity and any of the neurocognitive measures.

Linear regression analysis indicated a highly significant and negative correlation in WCST total correct responses score and sexual orientation $(t=-3.554, \mathrm{P}=0.001$, adjusted $\mathrm{R}^{2}=0.127$ ). The anatomical connectivity strength between left PoCG and left SMG was significant and positively related to sexual orientation $(t=-2.096, \mathrm{P}=0.039$, adjusted $\left.\mathrm{R}^{2}=0.072\right)$. However, this connection was also shown to be negatively related to education $(t=0.223, \mathrm{P}=0.047$, adjusted $\mathrm{R}^{2}=0.072$ ) (Table 3).

\section{Discussion}

To our knowledge, this is the first study to associate measures of cognitive performance in homosexual men with graph theoretical-based measures of their anatomical connectome. Anatomical connectome analysis indicated that homosexual men shared similar global architecture with heterosexual men, suggesting that overall brain structure may not be powerfully influenced by sexual orientation. Additionally, sexual orientation and experiences did not reshape the overall brain organization concerning topological efficiency and robustness. However, homosexual men displayed reduced connectivity between left PoCG and left SMG. Further linear regression analysis suggested that education was also a significant contributor to the connectivity strength between these two regions of the brain. While homosexual men also performed less well on the WCST. However, these cognitive measures in the homosexual men did not correlate significantly with their measures of connectivity.

Human cognitive functioning is generated by integrating information processes in functionally specialized areas. Graph theory posits that the brain is divided into several functionally independent nodes, and previous neuroimaging studies in healthy participants have shown that the connectivity among these nodes exhibits a smallworld property. This global feature is vital for supporting cognition and behavior and may alter in different biological conditions. In this study, the cognitive profiles associated with sexual orientation were similar to those reported in prior studies (3-5). The WCST is a neuropsychological test of "set-shifting", or flexibility in the face of changing schedules of reinforcement $(38,39)$. Performance on executive function tests such as WCST requires inhibiting responses to unnecessary information (40). Our findings revealed that homosexual men performed less well on the WCST compared to heterosexual men. This test is also 
Table 2 Comparison of neuropsychological test performance between homosexual and heterosexual men

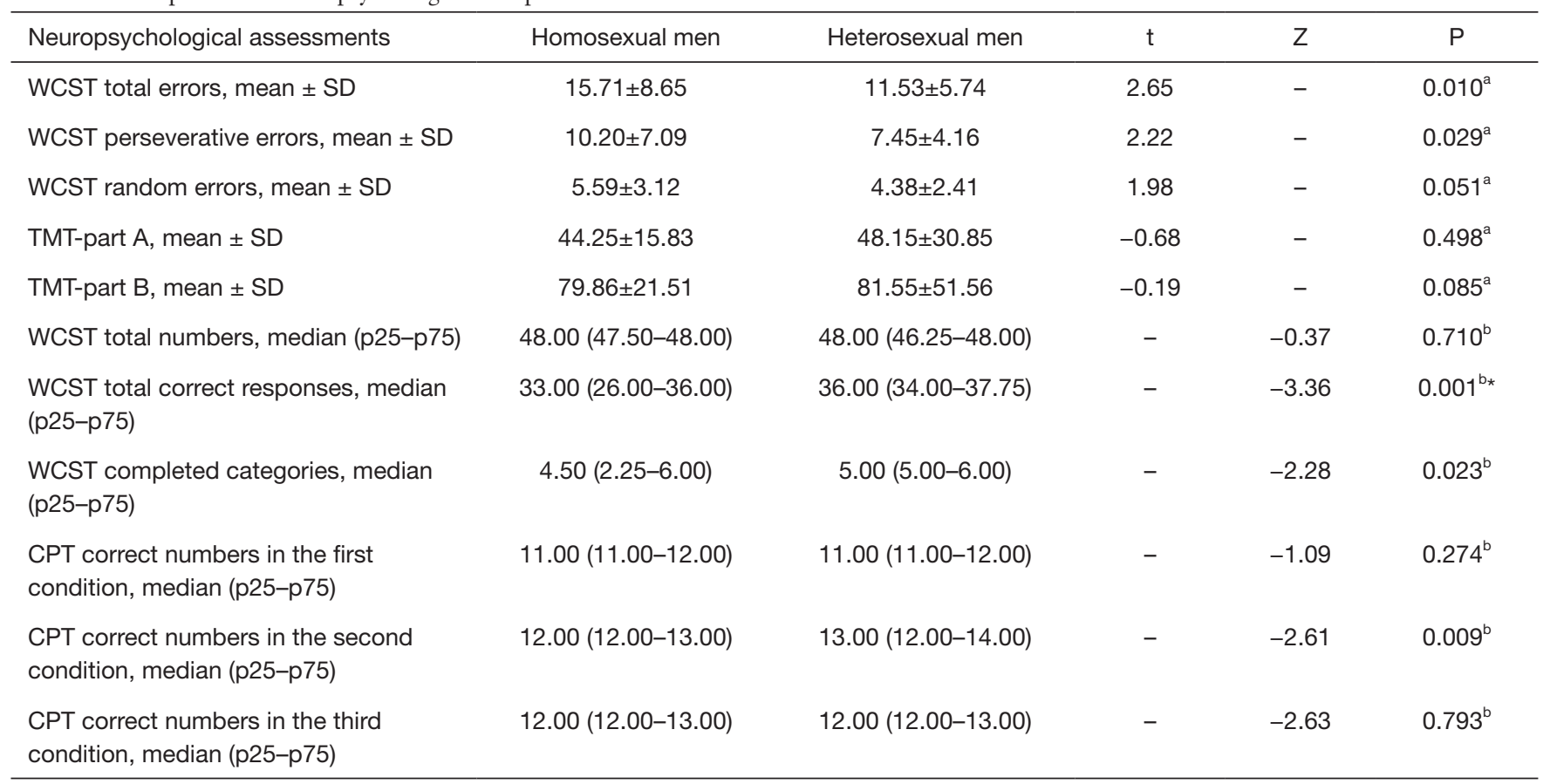

p25: 25th percentile; p75: 75th percentile. ${ }^{a}$, two-sample $t$-test; ${ }^{b}$, Mann-Whitney $U$ test; ${ }^{*}$, significant for $\mathrm{P}<0.004$, Bonferroni-corrected for multiple comparisons. WCST, Wisconsin Card Sorting Test; TMT, Trail-Making Test; CPT, Continuous Performance Test.

Table 3 Linear regression models for the association between neuropsychological assessments, topological features, and sexual orientation, and other clinical characteristics

\begin{tabular}{|c|c|c|c|c|c|c|c|c|c|c|}
\hline \multirow{3}{*}{ Variables } & \multicolumn{5}{|c|}{$\begin{array}{c}\text { Model } 1 \text { dependent variable: WCST total correct } \\
\text { responses score }\end{array}$} & \multicolumn{5}{|c|}{$\begin{array}{l}\text { Model } 2 \text { dependent variable: anatomical } \\
\text { connectivity strength }\end{array}$} \\
\hline & \multicolumn{2}{|c|}{$\begin{array}{l}\text { Unstandardized } \\
\text { coefficients }\end{array}$} & \multirow{2}{*}{$\begin{array}{c}\begin{array}{c}\text { Standardized } \\
\text { coefficients }\end{array} \\
\beta\end{array}$} & \multirow[t]{2}{*}{$t$} & \multirow[t]{2}{*}{$P$} & \multicolumn{2}{|c|}{$\begin{array}{l}\text { Unstandardized } \\
\text { coefficients }\end{array}$} & \multirow{2}{*}{$\begin{array}{c}\begin{array}{c}\text { Standardized } \\
\text { coefficients }\end{array} \\
\beta\end{array}$} & \multirow[t]{2}{*}{$t$} & \multirow[t]{2}{*}{$\mathrm{P}$} \\
\hline & $\beta$ & Std. error & & & & $\beta$ & Std. error & & & \\
\hline Constant & 35.580 & 4.032 & - & 8.828 & $<0.001^{*}$ & 0.314 & 0.027 & - & 11.605 & $<0.001^{*}$ \\
\hline Age, years & -0.164 & 0.118 & -0.149 & -1.383 & 0.170 & $-8.44 \mathrm{E}-5$ & 0.001 & -0.012 & -0.106 & 0.916 \\
\hline $\begin{array}{l}\text { Education, } \\
\text { years }\end{array}$ & 0.305 & 0.232 & 0.140 & 1.314 & 0.193 & 0.003 & 0.002 & 0.223 & 0.223 & $0.047^{\star}$ \\
\hline
\end{tabular}

*, significant for $\mathrm{P}<0.05$. WCST, Wisconsin Card Sorting Test.

considered a measure of executive functioning primarily due to its reported sensitivity to frontal lobe dysfunction $(41,42)$. However, it has been suggested that executive functions are regulated by the prefrontal regions of the frontal lobes $(43,44)$, but both frontal and non-frontal brain regions, including the SMG and PoCG, are necessary for intact executive functions (45).
The SMG is a vital part of the ventral parietal cortex (VPC) that supports attentional processes (46). Many functional MRI studies have shown that the VPC is involved in attention reorienting to sensory stimuli. The SMG, located in the temporo-parietal junction (TPJ), is strongly connected with the ventrolateral prefrontal cortex (VLPFC) and occipito-temporal regions (47), consistent 
with previous findings that the TPJ and VLPFC tend to coactivate in attention-reorienting studies $(48,49)$. Another study demonstrated the importance of SMG in the topdown control of selective attention (50). Transcranial magnetic stimulation (TMS) of the SMG impairs spatial attention only, whereas TMS of the anterior intraparietal sulcus disrupts spatial and feature-based attention (50). These effects are likely to derive from distinct mechanisms of topdown control between parietal and occipital regions (51).

The PoCG is also a vital part of the parietal lobe and is believed to play a significant role in visuomotor coordination. It has also been linked to a wide variety of high-level processing tasks $(45,52)$. For example, as the somatosensory association cortex, it plays a critical role in visuomotor attention and working memory, as well as visuospatial processing (52). Furthermore, the role of the SMG is consistent with the role of the superior longitudinal fasciculus in executive function, as this fiber tract connects frontal and parietal regions (53). The pioneering work of Catani (54) identified intrinsic connections between PoCG and SMG as the second prominent intraparietal tract. This is part of the arcuate fasciculus, a lateral associative bundle composed of long and short fibers connecting the perisylvian cortex of the frontal, parietal, and temporal lobes. Altered white matter hyperintensities related structural connectivity in left PoCG and SMG was found to be associated with poor attention set-shifting (55). Interestingly, a study reported that cortical thickness of left PoCG and SMG were increased after an arts education program, and the changes of the cortical thickness were associated with the performance of WCST (56). In line with the previous findings, our results also indicate that education significantly contributed to the connectivity strength between left PoCG and SMG besides sexual orientation.

The present study has shown that executive function differed between homosexual and heterosexual men, suggesting a link with sexual orientation. However, the overall similarity in global brain architecture was observed between homosexual and heterosexual men, with only reduced connectivity between left PoCG and left SMG, which was also influenced by education. These results indicate new perspectives in understanding the neural basis of male sexual orientation.

Some limitations of our study should be noted. Firstly, as an exploratory study, most of our findings of the anatomical connectome would not survive strict correction for multiple comparisons, although most connectomic studies share this limitation. Further validation in an independent sample is needed. Secondly, the variation in characteristics of homosexual participants, including their intelligence quotient, social extraversion, and differing Kinsey Scale scores, could influence the interpretation of findings in the correlation analyses. Lastly, heterosexual women were not included in this study, precluding the possibility of assessing whether brain and cognitive features in homosexual men were similar in pattern to those in heterosexual women. Above all, we have shown the differences in cognitive capacities and the anatomical connectome of large-scale brain networks between homosexual and heterosexual men, suggesting new perspectives to understanding this biological basis of male sexual orientation.

\section{Acknowledgments}

Funding: This work was supported by the National Natural Science Foundation of China (81101013 and 81671357 to SHH, 81171273 to KW, 81401400 to GJJ and 81471734 to DRX); Zhejiang Provincial Natural Science Foundation of China (LY14H180002 to SHH); National Clinical Research Center for Mental Health Disorders (2015BAI13B02); the Key Project of National Natural Science Foundation of China (91432301 and 91232717 to KW); National Basic Research Program of China (2015CB856405, 2012CB720704, and 2011CB707805 to KW); and Anhui Collaborative Innovation Center of Neuropsychiatric Disorder and Mental Health.

\section{Footnote}

Conflicts of Interest: All authors have completed the ICMJE uniform disclosure form (available at http://dx.doi. org/10.21037/qims-19-821b). The authors have no conflicts of interest to declare.

Ethical Statement: The study was approved by the Ethics Committee of the First Affiliated Hospital, Zhejiang University School of Medicine, and was carried out following the approved guidelines. Each participant was informed of the details about the study and provided written consent. The patient also consented to the publication of this study and any accompanying images. A copy of the written consent is available for review by the Editor-inChief of this journal.

Open Access Statement: This is an Open Access article 
distributed in accordance with the Creative Commons Attribution-NonCommercial-NoDerivs 4.0 International License (CC BY-NC-ND 4.0), which permits the noncommercial replication and distribution of the article with the strict proviso that no changes or edits are made and the original work is properly cited (including links to both the formal publication through the relevant DOI and the license). See: https://creativecommons.org/licenses/by-nc-nd/4.0/.

\section{References}

1. Marazziti D, Consoli G, Picchetti M, Carlini M, Faravelli L. Cognitive impairment in major depression. Eur J Pharmacol 2010;626:83-6.

2. Smith EE, Jonides J. Storage and executive processes in the frontal lobes. Science 1999;283:1657-61.

3. McCormick CM, Witelson SF. A cognitive profile of homosexual men compared to heterosexual men and women. Psychoneuroendocrinology 1991;16:459-73.

4. Sanders G, Ross-Field L. Sexual orientation and visuospatial ability. Brain Cogn 1986;5:280-90.

5. Rahman Q, Abrahams S, Wilson GD. Sexual-orientationrelated differences in verbal fluency. Neuropsychology 2003;17:240-6.

6. Rahman Q, Andersson D, Govier E. A specific sexual orientation-related difference in navigation strategy. Behav Neurosci 2005;119:311-6.

7. Tuttle GE, Pillard RC. Sexual orientation and cognitive abilities. Arch Sex Behav 1991;20:307-18.

8. Hamer DH, Hu S, Magnuson VL, Hu N, Pattatucci AM. A linkage between DNA markers on the X chromosome and male sexual orientation. Science 1993;261:321-7.

9. Hu S, Pattatucci AM, Patterson C, Li L, Fulker DW, Cherny SS, Kruglyak L, Hamer DH. Linkage between sexual orientation and chromosome $\mathrm{Xq} 28$ in males but not in females. Nat Genet 1995;11:248-56.

10. Mustanski BS, Dupree MG, Nievergelt CM, Bocklandt S, Schork NJ, Hamer DH. A genomewide scan of male sexual orientation. Hum Genet 2005;116:272-8.

11. Ellis L, Ames MA. Neurohormonal functioning and sexual orientation: a theory of homosexuality-heterosexuality. Psychol Bull 1987;101:233-58.

12. Blanchard R. Quantitative and theoretical analyses of the relation between older brothers and homosexuality in men. J Theor Biol 2004;230:173-87.

13. Bullmore ET, Bassett DS. Brain graphs: graphical models of the human brain connectome. Annu Rev Clin Psychol 2011;7:113-40.
14. McFadden D, Pasanen EG. Comparison of the auditory systems of heterosexuals and homosexuals: clickevoked otoacoustic emissions. Proc Natl Acad Sci U S A 1998;95:2709-13.

15. Savic I, Lindstrom P. PET and MRI show differences in cerebral asymmetry and functional connectivity between homo- and heterosexual subjects. Proc Natl Acad Sci U S A 2008; 105:9403-8.

16. Kinnunen LH, Moltz H, Metz J, Cooper M. Differential brain activation in exclusively homosexual and heterosexual men produced by the selective serotonin reuptake inhibitor, fluoxetine. Brain Res 2004;1024:251-4.

17. Hu SH, Wei N, Wang QD, Yan LQ, Wei EQ, Zhang MM, Hu JB, Huang ML, Zhou WH, Xu Y. Patterns of brain activation during visually evoked sexual arousal differ between homosexual and heterosexual men. AJNR Am J Neuroradiol 2008;29:1890-6.

18. Kagerer S, Klucken T, Wehrum S, Zimmermann M, Schienle A, Walter B, Vaitl D, Stark R. Neural activation toward erotic stimuli in homosexual and heterosexual males. J Sex Med 2011;8:3132-43.

19. Paul T, Schiffer B, Zwarg T, Kruger TH, Karama S, Schedlowski M, Forsting M, Gizewski ER. Brain response to visual sexual stimuli in heterosexual and homosexual males. Hum Brain Mapp 2008;29:726-35.

20. Ponseti J, Bosinski HA, Wolff S, Peller M, Jansen O, Mehdorn HM, Buchel C, Siebner HR. A functional endophenotype for sexual orientation in humans. Neuroimage 2006;33:825-33.

21. He Y, Evans A. Graph theoretical modeling of brain connectivity. Curr Opin Neurol 2010;23:341-50.

22. Bullmore E, Sporns O. Complex brain networks: graph theoretical analysis of structural and functional systems. Nat Rev Neurosci 2009;10:186-98.

23. Girvan M, Newman ME. Community structure in social and biological networks. Proc Natl Acad Sci U S A 2002;99:7821-6.

24. Nelson HE. A modified card sorting test sensitive to frontal lobe defects. Cortex 1976;12:313-24.

25. Dehaene S, Changeux JP. The Wisconsin Card Sorting Test: theoretical analysis and modeling in a neuronal network. Cereb Cortex 1991;1:62-79.

26. Homack S, Riccio CA. Conners' Continuous Performance Test (2nd ed.; CCPT-II). J Atten Disord 2006;9:556-8.

27. Reitan RM. The relation of the trail making test to organic brain damage. J Consult Psychol 1955;19:393-4.

28. Cui Z, Zhong S, Xu P, He Y, Gong G. PANDA: a pipeline toolbox for analyzing brain diffusion images. Front Hum 
Neurosci 2013;7:42.

29. Basser PJ, Mattiello J, LeBihan D. MR diffusion tensor spectroscopy and imaging. Biophys J 1994;66:259-67.

30. Mori S, Crain BJ, Chacko VP, van Zijl PC. Threedimensional tracking of axonal projections in the brain by magnetic resonance imaging. Ann Neurol 1999;45:265-9.

31. Zhang Z, Liao W, Chen H, Mantini D, Ding JR, Xu Q, Wang Z, Yuan C, Chen G, Jiao Q, Lu G. Altered functional-structural coupling of large-scale brain networks in idiopathic generalized epilepsy. Brain 2011;134:2912-28.

32. Watts DJ, Strogatz SH. Collective dynamics of 'smallworld' networks. Nature 1998;393:440-2.

33. Wang JH, Zuo XN, Gohel S, Milham MP, Biswal BB, He Y. Graph theoretical analysis of functional brain networks: test-retest evaluation on short- and long-term resting-state functional MRI data. PLoS One 2011;6:e21976.

34. Liang X, Zou Q, He Y, Yang Y. Coupling of functional connectivity and regional cerebral blood flow reveals a physiological basis for network hubs of the human brain. Proc Natl Acad Sci U S A 2013;110:1929-34.

35. Zhong S, He Y, Gong G. Convergence and divergence across construction methods for human brain white matter networks: an assessment based on individual differences. Hum Brain Mapp 2015;36:1995-2013.

36. Ji GJ, Zhang Z, Xu Q, Wei W, Wang J, Wang Z, Yang F, Sun K, Jiao Q, Liao W, Lu G. Connectome Reorganization Associated With Surgical Outcome in Temporal Lobe Epilepsy. Medicine (Baltimore) 2015;94:e1737.

37. Stuhrmann A, Dohm K, Kugel H, Zwanzger P, Redlich R, Grotegerd D, Rauch AV, Arolt V, Heindel W, Suslow T, Zwitserlood P, Dannlowski U. Mood-congruent amygdala responses to subliminally presented facial expressions in major depression: associations with anhedonia. J Psychiatry Neurosci 2013;38:249-58.

38. Berg EA. A simple objective technique for measuring flexibility in thinking. J Gen Psychol 1948;39:15-22.

39. Monchi O, Petrides M, Petre V, Worsley K, Dagher A. Wisconsin Card Sorting revisited: distinct neural circuits participating in different stages of the task identified by event-related functional magnetic resonance imaging. J Neurosci 2001;21:7733-41.

40. Potter LM, Grealy MA. Aging and inhibitory errors on a motor shift of set task. Exp Brain Res 2006;171:56-66.

41. Goldberg E, Bougakov D. Neuropsychologic assessment of frontal lobe dysfunction. Psychiatr Clin North Am 2005;28:567-80, 78-9.

42. Quan M, Lee SH, Kubicki M, Kikinis Z, Rathi Y, Seidman
LJ, Mesholam-Gately RI, Goldstein JM, McCarley RW, Shenton ME, Levitt JJ. White matter tract abnormalities between rostral middle frontal gyrus, inferior frontal gyrus and striatum in first-episode schizophrenia. Schizophr Res 2013;145:1-10.

43. Chen YC, Zhang H, Kong Y, Lv H, Cai Y, Chen H, Feng Y, Yin X. Alterations of the default mode network and cognitive impairment in patients with unilateral chronic tinnitus. Quant Imaging Med Surg 2018;8:1020-9.

44. Zhang L, Zhang L, Xue F, Yue K, Peng H, Wu Y, Sha O, Yang L, Ding Y. Brain morphological alteration and cognitive dysfunction in multiple system atrophy. Quant Imaging Med Surg 2018;8:1030-8.

45. Alvarez JA, Emory E. Executive function and the frontal lobes: a meta-analytic review. Neuropsychol Rev 2006;16:17-42.

46. Mesulam MM. A cortical network for directed attention and unilateral neglect. Ann Neurol 1981;10:309-25.

47. Daselaar SM, Fleck MS, Cabeza R. Triple dissociation in the medial temporal lobes: recollection, familiarity, and novelty. J Neurophysiol 2006;96:1902-11.

48. Corbetta M, Shulman GL. Control of goal-directed and stimulus-driven attention in the brain. Nat Rev Neurosci 2002;3:201-15.

49. Corbetta M, Patel G, Shulman GL. The reorienting system of the human brain: from environment to theory of mind. Neuron 2008;58:306-24.

50. Schenkluhn B, Ruff CC, Heinen K, Chambers CD. Parietal stimulation decouples spatial and feature-based attention. J Neurosci 2008;28:11106-10.

51. Saalmann YB, Pigarev IN, Vidyasagar TR. Neural mechanisms of visual attention: how top-down feedback highlights relevant locations. Science 2007;316:1612-5.

52. Wanchai. Cortical Functions: Reference. Hong Kong: Trans Cranial Technologis Ltd., 2012.

53. Sheline YI, Price JL, Vaishnavi SN, Mintun MA, Barch DM, Epstein AA, Wilkins CH, Snyder AZ, Couture L, Schechtman K, McKinstry RC. Regional white matter hyperintensity burden in automated segmentation distinguishes late-life depressed subjects from comparison subjects matched for vascular risk factors. Am J Psychiatry 2008;165:524-32.

54. Catani M, Robertsson N, Beyh A, Huynh V, de Santiago Requejo F, Howells H, Barrett RLC, Aiello M, Cavaliere C, Dyrby TB, Krug K, Ptito M, D'Arceuil H, Forkel SJ, Dell'Acqua F. Short parietal lobe connections of the human and monkey brain. Cortex 2017;97:339-57.

55. Respino M, Jaywant A, Kuceyeski A, Victoria LW, 
Hoptman MJ, Scult MA, Sankin L, Pimontel M, Liston C, Belvederi Murri M, Alexopoulos GS, Gunning FM. The impact of white matter hyperintensities on the structural connectome in late-life depression: Relationship to executive functions. Neuroimage Clin 2019;23:101852.
56. Park S, Lee JM, Baik Y, Kim K, Yun HJ, Kwon H, Jung YK, Kim BN. A Preliminary Study of the Effects of an Arts Education Program on Executive Function, Behavior, and Brain Structure in a Sample of Nonclinical SchoolAged Children. J Child Neurol 2015;30:1757-66.

Cite this article as: Wang Z, Hu JB, Ji GJ, Xu DR, Wang DD, Xi CX, Hu CC, Lu J, Du YL, Lu QQ, Huang TT, Lai JB, Chen JK, Zhou WH, Wei N, Xu Y, Wang K, Hu SH. Executive function and its relation to anatomical connectome in homosexual and heterosexual men. Quant Imaging Med Surg 2020;10(10):1973-1983. doi: 10.21037/qims-19-821b 